\title{
13 Neue editorische Ansätze und digitale tools - die philologische Revolution
}

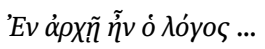

Evangelium nach Johannes 1,1

\subsection{Neue philologische Methoden als Faktoren verbesserter Editionen}

Es mag im ersten Moment überraschen, dass in den letzten Dekaden nicht nur technische Neuerungen, sondern auch viele genuin papyrologischphilologische Neuansätze einen entscheidenden Beitrag zu verbesserten Editionen geleistet haben. Die wichtigsten Entwicklungen und Methoden seien im Folgenden skizziert.

\subsubsection{Autopsie der Papyri und Transkription}

Janko (2016) hat eine ansprechende Anleitung für das Lesen und Edieren eines Herkulanischen Papyrus verfasst - mit einzelnen Arbeitsschritten vom Beschaffen von Arbeitsmaterial und der Anmeldung in der Officina bis hin zur Erstellung und Revision eines Manuskripts. ${ }^{163}$ Er empfiehlt zunächst eine handschriftliche Transkription anhand der MSI anzufertigen und diese anschließend am Original zu verifizieren. Der Ansatz und die Reihenfolge der vorgeschlagenen Arbeitsschritte sind stimmig, aber nicht sinnvoll auf alle Einzelfälle übertragbar. Jeder Papyrologe hat eine eigene, im Detail abweichende Herangehensweise entwickelt, die gewisse Vor- und Nachteile zeitigt. Manche Forscher zeichnen die Papyri erst vom Original ab, teils auf größeren Bögen im Format der cornici („piece mapping“), ${ }^{164}$ teils kolumnenweise im DIN A4-Format. Durch das Abzeichnen der Papyri am Original (und nicht durch das Abzeichnen der MSI) erhält man einen besseren Eindruck von der Arbeit der disegnatori und kann einige Fehler oder Eigenarten in den Disegni, etwa das unbewusste „Normieren“ von Buchstabenformen oder auch die falsche „Distanzmessung“ bei großen

163 Janko, How to read (wie Anm. 5), 119-134.

164 Diese Methode wurde von Graziano Ranocchia eingeführt und in seinen Projekten verschiedentlich angewandt, um die Stratigraphie der Papyri besser studieren zu können und Lagen einfacher zurückzuversetzen. 
Lücken, besser nachvollziehen. Oftmals arbeitet man „parallel“ mit den MSI (am Laptop) und dem Original. Manche Papyrologen übertragen die Beobachtungen auch direkt in den Computer. Man vergleicht (Zeile für Zeile) die MSI mit dem Original und prüft, ob die MSI fälschlicherweise Tinte anzeigen, wo im Original nur Risse oder Löcher zu sehen sind. Ferner versucht man eindeutig lesbare Buchstaben auf den MSI im Original zumindest ansatzweise zu erkennen oder zu erahnen. Ist dies nicht möglich, muss man akzeptieren, dass die MSI mehr als das menschliche Auge „sehen“ (und behandelt die Buchstaben in der Edition als wären sie im Original lesbar). Die cornici müssen für das Lesen am Original regelmäßig vorsichtig mit beiden Händen um einige Grad aus der Horizontalen herausgedreht werden. Für das Lesen und folglich auch Transkribieren der Herkulanischen Papyri benötigt man - ganz unwissenschaftlich gute Augen.

Meist verfassen die Papyrologen anhand der MSI oder eigener Abzeichnungen ein vorläufiges diplomatisches Transkript (oder ein artikuliertes Transkript bzw. einen Zwitter) und prüfen dieses (insbesondere eigene Ergänzungen) auf Kompatibilität mit dem Original. Die eigentliche philologische Denk- bzw. Puzzlearbeit findet im Wesentlichem außerhalb der Officina bzw. außerhalb Neapels vor dem PC oder den Aufzeichnungen/Transkripten statt, aber der Prozess des Lesens der Papyri (das Erkennen von Tinte) ist nicht immer trennscharf von der Philologie (möglichen Ergänzungen) zu scheiden. Die Herausgeber testen ihre philologischen Hypothesen am Original und oftmals führen lexikalische/philologische Notwendigkeiten auch zu einer Korrektur der ersten (vorläufigen) Transkription, d.h. des vermeintlich Gelesenen. Bei der Arbeit am Original ist das Erkennen von Lagen von besonderer Bedeutung (siehe 13.1.6). Einige Papyrologen fügen dem artikulierten (= literarischen) Transkript heute mehr oder weniger ausführliche Spurenbeschreibungen bei, während andere Papyrologen diplomatische Transkripte mit Spurenbeschreibung in Reinform erstellen. ${ }^{165}$ In meinem Apollodor-Buch ${ }^{166}$ und in der künftigen Gesamtausgabe des Index Academicorum streb(t)e ich bezüglich Spurenbeschreibung und diplomatischem Transkript eine gewisse Konvergenz mit der Darstellung in der The Oxyrhynchus Papyri-Serie an. Prinzipiell wurden die philologischpapyrologischen Angaben in Ausgaben der letzten zwei Jahrzehnte durch die Möglichkeiten moderner Textverarbeitungsprogramme immer genauer und komplexer (besonders im Hinblick auf Lagen und Disegni). Diese Entwicklung

165 Ein echtes diplomatisches Transkript wurde erstmals von Holger Essler, „Falsche Götter bei Philodem (Di III Kol. 8, 5 - Kol. 10, 6),“ CErc 39 (2009): (161-205) angefertigt.

166 Vgl. Fleischer, Apollodorus' Chronica (wie Anm. 112). 
ist an sich begrüßenswert und war auch ein „,versteckter“ Treiber vieler Verbesserungen, aber es besteht die Gefahr, dass durch ein Zuviel an papyrologischer Information die Lesbarkeit für Nicht-Fachleute negativ beeinträchtigt wird. ${ }^{167}$ Es ist essentiell, dass man sich beim Lesen der Papyri nicht unbewusst von philologischen Überlegungen oder Intentionen leiten lässt, welche den Spuren im Papyrus zuwiderlaufen. Eine objektive Transkription Herkulanischer Papyri ist wegen der Karbonisation mit ihren mannigfaltigsten Schwarzschattierungen ein schwieriges Unterfangen, erfordert viel Zeit und ist dennoch nur selten absolut „objektiv“. In einigen Fällen haben wir es bei der Beschreibung der Spuren faktisch mit gediegenen Wahrscheinlichkeitsaussagen zu tun. Auch üben frühere Editionen eine gewisse psychologisch-normative Kraft aus und verleiten häufig dazu, der vermeintlichen Autorität leichtfertig $\mathrm{zu}$ vertrauen und bisherige Lesungen im Papyrus erkennen zu wollen - auch wenn sie objektiv nicht mit den Spuren im Einklang sind. Die Rekonstruktion nur weniger Zeilen Herkulanischer Papyri kann mehrere Wochen in Anspruch nehmen, da wie in einem Kreuzworträtsel oder Schachspiel verschiedene Kombinationen oder Pfade, d.h. lexikalische Varianten, für die Lücken ausgetestet werden müssen und die syntaktische Grundstruktur oftmals schwer zu erkennen ist (siehe 14).

\subsubsection{Archivarbeit in der Officina}

Bei der Katalogisierung der Papyri und dem Kleben auf die cornici im 18. und 19. Jahrhundert kam es mitunter zu heilloser Unordnung und chaotischer Nummernzuteilung - teils ist die Schuld in Unzulänglichkeiten der Verantwortlichen, teils in widrigen externen Umständen zu suchen. Stücke ein und derselben Rolle sind heute unter verschiedenen Inventarnummern $\mathrm{zu}$ finden - oder eben auch nicht zu finden. Bisweilen gehören über zehn PHerc.-Nummern zur selben Rolle, wobei auf einigen cornici bzw. unter einigen Nummern die Teile von mehreren verschiedenen Papyri zusammengeklebt wurden. Durch akribische Archivarbeit in den Unterlagen der Officina dei papiri hat man in Verbindung mit anderen Kriterien in vielen Fällen wieder zusammenführen können, was zusammengehört. Insbesondere ist die Entdeckung des ältesten Inventars

167 Durch eine Trennung von diplomatischem und artikuliertem Transkript (und keiner Kreuzung) kann eine Überfrachtung mit diakritischen Zeichen im artikulierten Transkript verhindert werden. 
der Papyri aus der Zeit Piaggios zu erwähnen, ${ }^{168}$ was verbunden mit neuen bibliometrischen Verfahren (13.1.4) oft eine Kalkulation der ursprünglichen Rollenlängen bzw. des verlorenen Textes erlaubt. Auch bezüglich der Zuordnung von Disegni, die manchmal irreführende, nicht mit dem Erhaltenen kompatible Nummern haben, konnten Einzelstudien mehrere Probleme lösen. Somit sind Beiträge zur „Geschichte der Officina“, etwa zur Entrollung, Abzeichnung und (Um)Lagerung, nicht unter der Rubrik „Wissenschaftsgeschichte“ oder belanglose „Sammlungsgeschichte“ abzuhaken, sondern haben sehr oft eine unmittelbare Relevanz für die Rekonstruktion der Papyri oder die Zuweisung von Fragmenten, also letztlich für die restitutio textus. Viele einschlägige Dokumente wurden nicht nur in der Officina selbst, sondern bspw. auch im Museo Archeologico Nazionale (früherer Aufbewahrungsort der Papyri) oder in Oxford wiedergefunden. Herausgeber versuchen die Geschichte ihres Papyrus (Entrollung, ggf. mit scorzatura parziale, Einzelteile, Abzeichnungen) sehr sorgfältig zurückzuverfolgen, um sich eine optimale Basis für die Rollenrekonstruktion und Edition $\mathrm{zu}$ verschaffen. ${ }^{169}$ Grundlegende Archivarbeit vieler Forscher während der letzten Jahre hat für kommende Neuausgaben eine bessere Ausgangslage geschaffen.

\subsubsection{Delattre-Obbink-Methode (Reihenfolge der scorze)}

Auch wenn ab der Zeit von Piaggio schon bald keine scorzatura totale mehr durchgeführt wurde (siehe 4.1), war es doch oft noch nötig, die äußeren Lagen des Papyrus abzutrennen (siehe 4.2), um an den relativ gut ausrollbaren Mittelteil der Rollen (midollo) heranzukommen. Dabei entstanden oft (mehrere) Halbzylinder, welche von innen nach außen ausgekratzt wurden (scorzatura parziale), wobei die abgezeichneten Lagen (Fragmente bzw. scorze) zerstört wurden, um das nächste Blatt abzeichnen zu können. Die disegnatori nummerierten mit der Zahl 1 beginnend durch. Folglich ist das innerste Fragment mit 1 bezeichnet und die Fragmente erscheinen in numerisch inverser Reihenfolge, d.h. nicht in der Reihenfolge, in welcher sie im Papyrus relativ aufeinanderfol-

168 Vgl. David Blank und Francesca Longo Auricchio, „Inventari antichi dei Papiri Ercolanesi,“ CErc 34 (2004): (39-152). Weitere wichtige Beiträge mit Relevanz für die Textherstellung sind etwa Bassi, Piaggio e i primi tentativi (wie Anm. 36); Blank, „Reflections on Rereading

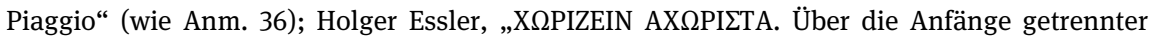
Aufbewahrung der Herkulanischen Papyri,“ CErc 40 (2010): (173-189).

169 Vgl. Janko, „How to read“ (wie Anm. 5), 134-137. 
gen. Die Herausgeber im 19. und 20. Jahrhundert dachten nicht weiter über die Nummerierung und den Prozess der Fragment-Gewinnung nach und nahmen natürlicherweise an, dass „Fragment 1“ auch das erste Fragment (am Anfang bzw. relativ vorne) im Papyrus war und Fragment 2, Fragment 3 usw. in der der Folge ihrer Nummerierung relativ aufeinander folgten. Somit wurden diese Fragmente in allen früheren Ausgaben in falscher Reihenfolge gelesen bzw. angeordnet! Erst in den 1990er Jahren erkannten Daniele Delattre und Dirk Obbink unabhängig voneinander, ${ }^{170}$ dass die Fragmente invers zu ihrer tatsächlichen Reihenfolge im Papyrus nummeriert sind und ,umgedreht“ werden müssen, was für das korrekte Extrapolieren von längeren Gedankengängen, Strukturen oder Argumentationsabschnitten entscheidend ist.

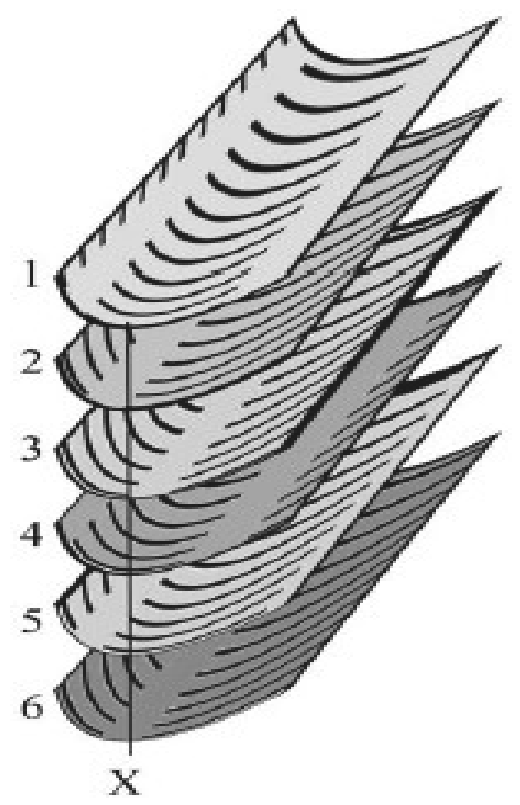

Abb. 48: Falsche Nummerierung der scorze - eigentlich ist Fragment 6 das „erste“ Fragment der Rolle

170 Vgl. Delattre, rouleaux d' Herculanum (wie Anm. 5), 116-119. Jedoch hat schon Adolf Schober, „Philodemi De pietate pars prior,“ CErc 18 (1988): (67-125) 67,70 Fragmente ohne tiefere Erläuterung im Sinne dieser Methode umgeordnet. 


\subsubsection{Neue mathematisch-bibliometrische Verfahren zur Rollenrekonstruktion}

Aufgrund der (Teil)Entrollung eines Papyrus zu verschiedenen Zeiten und aufgrund von Lücken zwischen Kolumnen oder cornici ist es oft nötig zu schätzen, wieviel Kolumnen (bzw. ob Kolumnen) in diesen Lücken oder zwischen Fragmenten verlorengegangen sind, was für die restitutio textus (Konjekturen) sehr wichtig ist. Holger Essler (2008) hat in einem grundlegenden Artikel zur mathematischen Rekonstruktion der Rollen frühere Erkenntnisse zusammengefasst, ausgearbeitet und systematische Berechnungsansätze vorgelegt. ${ }^{171}$ Ein Schlüsselelement der mathematischen Rekonstruktion sind die Wicklungen (Voluten) der Rolle, welche oftmals am aufgerollten Originalpapyrus gut abgemessen werden können. Wiederkehrende Strukturen - teils ziehen sich StreifenStrukturen (,Sektionen“) durch weite Teile des Papyrus - sind hier bedeutsam. ${ }^{172}$ Das Prinzip der Wicklungen besteht darin, dass die Breite der Wicklungen der Rollen von innen nach außen jeweils konstant um etwa 1-2 mm pro Wicklung zunimmt. ${ }^{173}$ Die Kenntnis des genauen Wicklungsumfangs (= Wicklungsbreite im aufgerollten Zustand) ist für das chirurgisch präzise Platzieren von beim Aufrollprozess falsch hängengebliebenen Lagen (Sovrapposti und Sottoposti) notwendig. Ferner erlaubt das Wissen darüber, (wieviel) Text zwischen einzelnen Fragmenten des Papyrus ausgefallen ist (oft im Zusammenhang mit der „Entrollgeschichte“ des Papyrus bzw. der verschiedenen Papyrusteile, siehe 13.1.2), eine genaue oder ungefähre Rekonstruktion der Länge (von Abschnitten) und Kolumnenanzahl. Hier sind nicht nur die zur Verfügung stehenden messbaren Variablen (etwa Wicklungsbreite, zählbare Kolumnen, ursprünglicher Rollendurchmesser und die Breite der Kollemata), sondern auch „textuelle“ Variablen wie etwa stichometrische Zeichen und stichometrische Angaben am Ende der Rolle relevant. Diese Variablen haben eine gewisse Interdependenz und helfen bei der Rekonstruktion der Rolle bzw. von Rollenabschnitten und können zugleich Rekonstruktionshypothesen plausibilisieren

171 Vgl. Essler, „Rekonstruktion von Papyrusrollen“ (wie Anm. 148). Unter den früheren Arbeiten ist etwa Maria Nardelli, „Ripristino topografico di sovrapposti e sottoposti in alcuni papiri ercolanesi,“ CErc 3 (1973): (104-115) mit Entdeckung des Prinzips der „sezioni“ zu erwähnen.

172 Wickelt man ein Blatt Papier (DIN A4) zu einer Rolle fest zusammen und wickelt diese nach einiger Zeit wieder auf, erkennt man schwache Streifen auf dem Papier, die im Abstand von einer Wicklung (Volute), teils auch Halbwicklungen (Semi-Voluten), zueinander liegen. Ähnliches ist bei den Papyri zu beobachten.

173 Essler, „Rekonstruktion von Papyrusrollen“ (wie Anm. 148), 286-287. 
oder falsifizieren. Diese Methoden wurden in der letzten Dekade von vielen Papyrologen für ihre Neueditionen erstmalig angewendet. Essler hat auch ein frei zugängliches excel-sheet für Kalkulationen entwickelt, ${ }^{174}$ wobei der jeweilige (mathematische) Weg zur Rekonstruktion auch bedingt vom Einzelfall abhängt (siehe 12) und man nicht immer schematisch vorgehen kann. Die zugrundeliegenden mathematischen Gedanken sind zwar nicht völlig trivial, aber auch nicht hochkomplex - Papyrologen sollten diese mit etwas Studium nachvollziehen können.

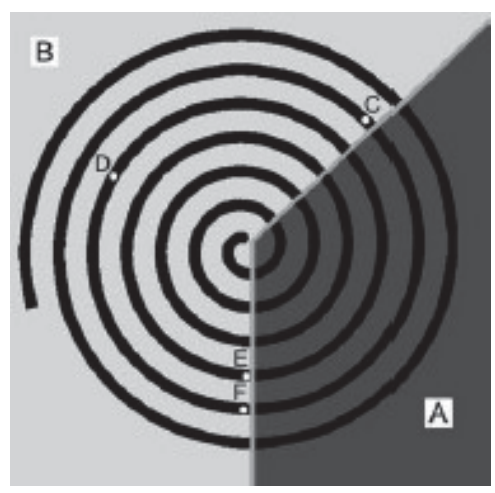

Abb. 49: Papyrusrolle als Archimedische Spirale

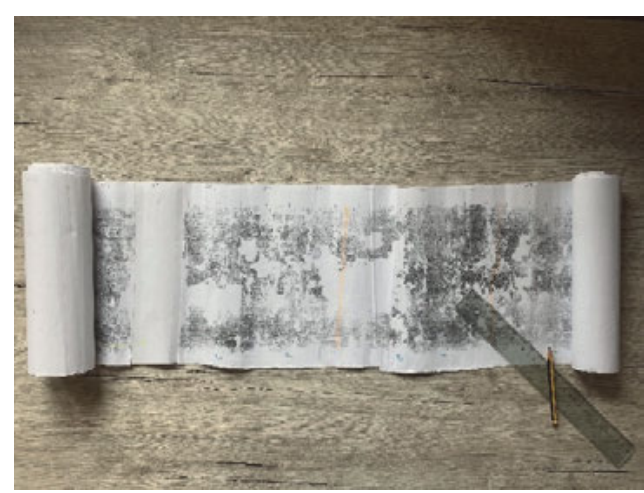

Abb. 50: Rollenmodell 1:1

\subsubsection{Rollenmodelle}

Unter Zuhilfenahme obiger Methoden erstellt man entweder ein physikalisches Rollenmodell im Maßstab 1:1 (Ausdrucken und Zusammenkleben der MSI/Disegni) oder ein virtuelles Modell am Computer. Solche Modelle sind keine ästhetischen Spielereien, sondern fast essentiell für das korrekte Platzieren von Sovra- und Sottoposti, gerade bei stratigraphisch herausfordernden Papyri. Ferner vermitteln die Modelle einen haptischen Überblick über Verlust und Lücken im Papyrus. In den Ausgaben werden Modelle der Rolle oder von Rollenteilen teils bildlich gedruckt, teils bibliometrische Daten in Tabellen zusammengefasst. Einige wichtige Elemente der mise en page sind: Länge und Höhe

174 Das excel-sheet kann auf http://epikur-wuerzburg.de/ heruntergeladen werden. 
der Rolle, Anzahl der Kolumnen, Kolumnenbreite, ${ }^{175}$ beschriebener Raum, Zeilen pro Kolumne, Wörter pro Zeile. In den Herkulanischen Papyri selbst steht am Rollenende öfters die Summe der oтíxoı, was aber nicht die tatsächlichen Zeilen, sondern „Normzeilen“ meint, die meist 1,5 bis 2 Zeilen im Papyrus entsprechen. Auch die Einzelblätter (Kollemata), aus denen die Rolle zusammengeklebt ist, sind manchmal von Interesse.

\subsubsection{Exaktere Rückplatzierung falscher Lagen - Sovrapposti und Sottoposti}

Wie bereits dargelegt, hängt die genaue Platzierung falscher Lagen mit der bibliometrischen Rekonstruktion der Rolle, d.h. der Breite der Wicklungen zusammen. ${ }^{176}$ Meist reden wir bei den falschen Lagen nicht über Stücke von mehreren Quadratzentimetern, sondern über winzige Fetzen, deren Fläche geringer als 1 $\mathrm{cm}^{2}$ (ein Daumennagel) ist. Sie sind in der Hügellandschaft der Papyri nicht immer einfach zu isolieren, da oft unklar ist, ob Risse oder Verwerfungen innerhalb derselben Ebene oder doch verschiedene Ebenen vorliegen. Erfreulicherweise ist zu konstatieren, dass in den letzten Jahren die Identifikation der Lagen mit größerer Akribie und Geduld vorgenommen wurde und viele Neulesungen ermöglichte. ${ }^{177}$ Früher verkannte man häufiger Lagen und las Buchstaben(teile) „in Reihe“, obwohl sie teils zu anderen Schichten gehörten, was zu einem Phantasietext führte, der nur durch den neuzeitlichen Aufrollprozess zustande gekommen war, aber nicht im antiken Papyrus zu lesen war. Das Identifizieren und Platzieren der Lagen, gerade von kleinsten Teilen, kann sehr ermüdend und zeitraubend sein. Mit verbesserten Mikroskopen und insbesondere dem virtuellen Ausschneiden und Rückversetzen der Lagen am Computer ist das Umordnen der Lagen nun um ein vieles einfacher und ersprießlicher. Nichtsdestotrotz sind einige Lagenidentifikationen oder Rückplatzierungen weiterhin nicht über alle Zweifel erhaben. Marzia D`Angelo hat kürzlich die Anforderun-

175 Der interkolumnare Raum (Kolumnenbreite+Interkolumnium) bzw. „Seitenbreite“ ist innerhalb eines jeden Herkulanischen Papyrus relativ konstant, vgl. Essler, „Rekonstruktion von Papyrusrollen“ (wie Anm. 148), 290.

176 Die Breite der Wicklungen im aufgerollten Papyrus entspricht dem Umfang im unaufgerollten Papyrus. Teils kann auch durch die lexikalisch zweifelsfreie Platzierung solcher Lagen auf die (unbekannte) Wicklungsbreite geschlossen werden.

177 Oft auch mit negativem Effekt: Manche Lesungen wurden durch nun „störende“ Buchstaben unmöglich. 
gen an eine spezielle Software für eine bessere und leichtere Rückplatzierung der Sovrapposti und Sottoposti skizziert. ${ }^{178}$

\subsubsection{Neue Editionsreihen - Formate und Ansätze (seit etwa 2000)}

Während die Scuola di Epicuro im letzten Viertel des 20. Jahrhundert fraglos die Rolle des „Platzhirsches“ unter den Herkulanischen Editionen einnahm, publizierten auch mehrere Wissenschaftler in anderen Reihen. $\mathrm{Zu}$ nennen ist etwa Gaisers Ausgabe des Index Academicorum (1988), in der mit viel Aufwand alle Disegni des Papyrus abgedruckt wurden und eine Mischung von Kolumnenform und zusammenhängender Übersetzung vorgenommen wurde. ${ }^{179}$ Die in den 90er-Jahren begonnene Reihe The Philodemus translation series kehrte zur Kolumnendarstellung des griechischen Textes (samt Apparat auf der linken Seite) zurück, ${ }^{180}$ wobei der griechische Text nochmals (etwas redundant) als Fließtext samt Übersetzung und Kommentar auf der rechten Seite steht; ${ }^{181}$ weitere Bände sind angekündigt. ${ }^{182}$ Einer reinen Kolumnenform bediente sich auch Delattre für die Ausgabe von Philodems De musica in Collection Des Universités De France. Serie Grecque (mit dem griechischen Text auf der rechten Seite). ${ }^{183}$ Mit griechischem Text links und englischer Übersetzung rechts erscheinen die Editionen in der Serie Writings from the Greco-Roman-World. ${ }^{184}$ Auch die Scuola di Epicuro ist

178 Marzia D“ Angelo, „Verso un software per la ricostruzione dei papiri ercolanesi con stratigrafia complessa,“ CErc 50 (2020): (161-162).

179 Gewisse Ähnlichkeit zur Darstellung in „Ricerche sui papiri Ercolanesi,“ (siehe Anm. 94).

180 So auch schon Tiziano Dorandi, Filodemo, Storia Dei Filosofi: La Stoà da Zenone a Panezio (PHerc. 1018) (Leiden, 1994).

181 Die Serie ist auch bekannt als (bzw. verbunden mit) The Philodemus translation project (oder: Philodemus project - siehe https://classics.ucla.edu/faculty-projects/philodemusproject/). Bisherige Ausgaben: Dirk Obbink, Philodemus on Piety: Critical Text with Commentary. Part 1 (Oxford, 1996); Richard Janko, Philodemus. On Poems (3 Bände) (Oxford, 20012010-2020). Blank und Hammerstaedt arbeiten an Ausgaben von Philodems De rhetorica. Das Format der Reihe wurde auch von Graziano Ranocchia, Aristone ,Sul modo di liberare dalla superbia' nel decimo libro,De vitiis‘ di Filodemo (Florenz, 2007) übernommen.

182 Aus der Aufstellung bei Delattre, rouleaux d'Herculanum (wie Anm. 5), 102-104 wird ersichtlich, dass viele angekündigte Übersetzungs- und Editionsvorhaben von diversen Gelehrten (faktisch) aufgegeben wurden.

183 Daniel Delattre, Philodeme de Gadara, Sur La Musique, Livre IV (2 Bände) (Paris, 2007).

184 Die Serie wird von der „Society of Biblical Literature“ herausgegeben. Erschienen sind bisher: Benjamin Henry, Philodemus. On Death (Atlanta, 2009); Voula Tsouna, Philodemus, On property management (Atlanta, 2012); Armstrong und McOsker, Philodemus. On Anger (wie Anm. 49). 
in den letzten Bänden zur Kolumnenform übergegangen. ${ }^{185}$ Innovative Editionen, die auch systematische diplomatische und literarische Transkripte mit Spurenbeschreibung einschlossen, wurden im Rahmen eines EU-Starting Grant (2009-2014) von Ranocchia erprobt. ${ }^{186}$

Generell fällt bei den meisten Neueditionen positiv auf, dass es im Vergleich zu früheren Dekaden oder Jahrhunderten mit Texteingriffen oder „ingeniösen“ Ergänzungen nicht mehr übertrieben wird - diese haben sich im Nachhinein nur zu oft als falsch herausgestellt und führten leider manchmal über Jahre hinweg zu irrigen Folgerungen oder Hypothesen. Auch werden seltener stillschweigend Korrekturen vorgenommen, indem Spuren im Papyrus gegen die Evidenz „zurechtgebogen“ oder „zurechtinterpretiert“ werden. ${ }^{187}$ Die Integration bzw. Berücksichtigung der Disegni erfolgt systematischer und oft geben die Editoren eine Art (kleines) „diplomatisches Transkript“, welches Lesarten verschiedener Quellen nennt und auch die Lagen erklärt. Ferner wird immer größerer Wert auf Anschaulichkeit gelegt (gerade bei den bibliometrischen Einleitungen), was viel zum Verständnis beiträgt. Auch die Kommentare profitieren von neuen digitalen tools (etwa für Parallelstellen).

\subsection{Digitale tools, Bilder und Datenbanken als Faktoren verbesserter Editionen}

\subsubsection{Digitale Bilder: Disegni, frühe (Bild)Ausgaben und MSI}

Die Oxforder Disegni sind heute vollständig digitalisiert und online zugänglich. ${ }^{188}$ Auch mit der Digitalisierung der Neapolitanischen Disegni wurde vor

185 Giuliana Leone, Epicuro, Sulla natura, Libro II. Edizione, traduzione e commento (Neapel, 2012); Federica Nicolardi, Filodemo. Il primo libro della Retorica (Neapel, 2018).

186 ERC project „Interactive edition and interpretation of various works by Epicurean and Stoic philosophers surviving at Herculaneum“ (PHerc), siehe http://www.pherc.eu/; vgl. auch Graziano Ranocchia, Testi stoici ed epicurei della collezione ercolanese (Rom, 2016).

187 Die Kollegen können heute durch die MSI rasch Lesungen (vorläufig) falsifizieren bzw. anzweifeln und müssen den Herausgebern nicht mehr „blind“ vertrauen, was diese dazu zwingt, Willkür oder Nachlässigkeit auf ein Mindestmaß zu reduzieren bzw. sich diese nicht mehr zu erlauben.

188 Am besten nutzt man den Link auf der Seite der Herculaneum Society (www.herculaneum.ox.ac.uk/papyri/online-resources), welche auf das mit der Universität Oxford verbundene „The Imaging Papyri Project“ bzw. eine entsprechende Seite führt. 
einigen Jahren begonnen. Viele Disegni sind bereits im Internet abrufbar. ${ }^{189}$ Letztlich gleichen die Neapolitanischen Disegni zu 95-99\% den entsprechenden Illustrationen in den 11 Bänden der collectio altera. ${ }^{190}$ Diese Bände sowie die Kupferstiche (nicht Editionen!) der collectio prior sind heute ebenfalls vollständig auf der Seite des Würzburger Zentrum für Epikureismusforschung einsehbar. ${ }^{191}$ Mehrere digitalisierte Bände der collectio prior lassen sich auch durch Suche im Internet finden. Die Multispektralbilder (MSI) der Herkulanischen Papyri sind bedauerlicherweise nicht online zugänglich, aber sie können bei der Brigham Young University (BYU) für interessierende PHerc.-Nummern angefragt werden. ${ }^{192}$

\subsubsection{Online-Katalog chartes}

$\mathrm{Zu}$ einem sehr nützlichen Tool hat sich auch der online-Katalog chartes entwickelt, welcher Informationen früherer Kataloge (siehe 7.4) kombiniert und für jede PHerc.-Nummer ein Bild, das Aufrolldatum und weitere Angaben enthält. ${ }^{193}$ Die von Gianluca Del Mastro verwaltete und vom CIPSE initiierte, in regelmäßigen Abständen aktualisierte Datenbank erschien bereits 2005 als CD und ist seit einigen Jahren online durchsuchbar. Für jeden Papyrus ist quasi umfassend die zugehörige Bibliographie gelistet, was die Datenbank zur umfangreichsten bibliographischen Ressource für Herkulanische Papyri macht. Die bibliographischen Verweise sind sehr präzise (genaue Seite, auf welcher der Papyrus/die Nummer in einem Beitrag erscheint); auch sind mitunter direkte Links zu den Beiträgen (pdfs) vorhanden. Man kann die Sammlung nach 11 Kriterien durchsuchen und auch kombinierte Suchen ausführen. Viele (statistische) Studien und mancherlei Erkenntnisse zur Zuordnung von Papyri (siehe 13.1.2) wurden erst durch den online-Katalog möglich.

189 Die Disegni findet man auf der Seite der Biblioteca Nazionale di Napoli (BNN): http://digitale.bnnonline.it/index.php?it/209/disegni-napoletani-dei-papiri-ercolanesi.

190 In der Officina sind auch die nicht digitalisierten Druckfahnen der collectio altera gelagert, welche aber nur von beschränktem Wert sind.

191 Auf der Seite sind ebenfalls The King's Book und in absehbarer Zeit die Davy Disegni (siehe 4.3) abrufbar.

192 HSI wurden bisher nur von PHerc. 1021 gemacht. Diese können in der Officina konsultiert werden. Für die MSI sollte man sich an Roger MacFarlane wenden; eine Genehmigung der Nationalbibliothek von Neapel ist nötig.

193 www.chartes.it. 


\subsubsection{Die Herkulanischen Texte online - THV, DCLP und TLG}

Ebenso wichtig wie der online-Katalog chartes ist der Thesaurus Herculanensium voluminum (THV), eine Volltextdatenbank (fast) aller Herkulanischen Papyri, welche mittlerweile in den Digital Corpus of Literary papyri (DCLP) integriert ist, was ihr Durchsuchen nach bestimmten Wörtern oder Wortkombinationen oder nach anderen Kriterien ermöglicht. ${ }^{194}$ Nachdem zunächst auf einer separaten Seite 26 Herkulanische Texte im Zuge einer Kooperation des CISPE mit der Universität Würzburg digitalisiert wurden (Del Mastro und Essler), begann 2013 die von Essler koordinierte systematische Erfassung aller Papyri der Sammlung (zurzeit 158 Texte von 277 Inventarnummern) und ihre Übertragung in den DCLP, so dass der THV als selbständiges Corpus praktisch im DCLP aufging. ${ }^{195}$ Eine übersichtliche Liste aller im DCLP erfassten Herkulanischen Papyri, Autoren und Werke findet man auf der Seite des Würzburger Zentrum für Epikureismusforschung. ${ }^{196}$ Für die Kodierung wurden die letzten Gesamtausgaben der Papyri genutzt, so dass viele spätere Neulesungen in Teilausgaben nicht im Text des DCLP erscheinen, welcher aber einen kleinen kritischen Apparat und andere nützliche Angaben bietet.

Ein weiteres, schon einige Jahrzehnte zur Verfügung stehendes wichtiges Hilfsmittel für die Textergänzung der Papyri ist der Thesaurus Linguae Graecae in digitaler Form (online-TLG bzw. früher der TLG auf CD-Rom), über welchen nach Wortteilen, Wörtern oder Kombinationen von Wörtern gesucht werden kann. In den TLG sind aber nicht alle Herkulanischen Texte aufgenommen.

Einige auch mit den Herkulanischen Papyri in Verbindungen stehende theoretische und technische Aspekte der Digitalisierung von Papyri wurden im Rahmen des „Ekdosis-Projektes“ erforscht. ${ }^{197}$ Das Grammatically Annotated Philodemus project zielt darauf ab, die Lexik, Grammatik und Syntax der Papyri mittels computergestützter Methoden zu analysieren. ${ }^{198}$

194 Der DCLP ist wiederum integraler Bestandteil der Seite http://www.papyri.info/, auf der diverse Corpora mit einem search-navigator durchsucht werden können.

195 Die alte Seite des THV (www.thvproject.it) existiert nicht mehr, vgl. Reggiani, Digital Papyrology (wie Anm. 136), 245-246 und Rodney Ast und Holger Essler, „Anagnosis, Herculaneum, and the Digital Corpus of Literary Papyri,“ in Digital Papyrology II (hg. von Nicola Reggiani; Berlin, 2018), (63-73).

196 http://epikur-wuerzburg.de/aktivitaeten/thv/.

197 Projekt: „Ekdosis: Digitizing literary and paraliterary papyri“ (2018-2020 - Holger Essler/Massimo Magnani/Nicola Reggiani).

198 Holger Essler und Daniel Riano Rufilanchas, „,Aristarchus X“ and Philodemus: Digital Linguistic Analysis of a Herculanean Text Corpus,“ in Proceedings of the 27th International 


\subsubsection{Bibliographien und wichtige Websites}

$\mathrm{Zu}$ Philodem findet man in Stanford Encyclopedia of Philosophy einen aktualisierten Beitrag von David Blank mit einer Übersicht zu Biographie, Werken, philosophischer und schriftstellerischer Bedeutung sowie Rezeption, der natürlicherweise engsten Bezug $\mathrm{zu}$ den Herkulanischen Papyri hat. ${ }^{199}$ Für Oxford Bibliographies Online besorgte Essler eine profunde Bibliographie zu Philodem mit besonderem Fokus auf den Editionen seiner Werke, während Benjamin Henry die Bibliographie für die Herkulanischen Papyri allgemein oblag. Beide Einträge haben einige Überschneidungen und sind für Literatur zu Herkulanischen Papyri der erste Anlaufpunkt. ${ }^{200}$

Nützlich ist auch die Website des Würzburger Zentrums für Epikureismusforschung. ${ }^{201}$ Hier finden sich neben den schon angesprochenen digitalen Ressourcen ein von Studenten immer weiterentwickeltes (nicht immer zitierfähiges) „Herkulaneum-Wiki“ sowie weitere Such-tools. Auf der Internetseite des CISPE können mittlerweile alle Artikel der CErc-Bände einzeln (gegen Gebühr) heruntergeladen werden. ${ }^{202}$ Auch ist zu vermerken, dass am Ende aller CErc-Bände gängige Abkürzungen für das Zitieren der Papyri zusammengetragen sind und unter „notiziario“ vermerkt ist, wer gerade an welchen Papyri arbeitet. Zwar haben diese Angaben keine juristische oder verbindliche Geltung, aber sie bieten eine Orientierungshilfe zur aktuellen Forschungssituation und können unnötige Parallelarbeiten vermeiden helfen. Auf der Seite der Herculaneum Society (9.3) sind neben den schon erwähnten Ressourcen noch einige andere hilfreiche

Congress of Papyrology 2013 (Warsaw) (hg. von Thomasz Derda/Adam Lajtar/Jakub Urbanik; Warschau; 2016), (491-501).

199 https://plato.stanford.edu/entries/philodemus/ (2013 erstellt, 2019 substantiell überarbeitet).

200 https://www.oxfordbibliographies.com. Die Bibliographie zu Philodem (,Philodemus of Gadara“) wurde 2019 aktualisiert, die für die Herkulanischen Papyri („Herculaneum Papyri“) zuletzt 2018. Ferner findet sich auch (nicht aktuell) eine Zusammenstellung von Philodems Werken auf https://www.herculaneum.ox.ac.uk/papyri/other-resources/books-herculaneum. Eine Bibliographie für lateinische Papyri Herkulaneums findet sich unter http://web.philo.ulg.ac.be/cedopal/les-papyrus-latins-dherculanum (Gabriel Nocchi Macedo).

201 https://epikur-wuerzburg.de/.

202 Die online-Verfügbarkeit der Zeitschrift Cronache Ercolanesi über einschlägige Elektronische Zeitschriftenkataloge ist leider noch immer ein Desiderat, dem hoffentlich in nicht zu ferner Zukunft Genüge getan wird, um eine breitere wissenschaftliche Rezeption der Forschung an den Herkulanischen Papyri zu gewährleisten. 
Links zu den Papyri und aktuelle Nachrichten $\mathrm{zu}$ technischen Fortschritten gesammelt. ${ }^{203}$

\subsection{Die Herkulanischen Papyri in den Medien}

Die archäologischen Stätten Pompejis und Herkulaneums ziehen Menschen seit mehr als 250 Jahren in ihren Bann und so verwundert es wenig, dass sie auch in den Medien und der Populärkultur recht präsent sind. Neufunde und Entwicklungen werden mit steter Regelmäßigkeit international in Nachrichten erwähnt. Die Papyri und die Bibliothek spielten in der medialen Rezeption lange Zeit eher eine Nebenrolle, erfuhren jedoch in den letzten zwei Jahrzehnten durch neue Bildgebungsverfahren und virtual unrolling eine erstaunliche Beachtung in Zeitung, Radio, TV und Internet. Nach kleineren Berichten im TV und Zeitungen (vornehmlich in Italien) im 20. Jahrhundert hat die Dokumentation „Out of the ashes: Recovering the lost library of Herculaneum“ (2003), welche zunächst auf DVD erhältlich war und nun auch online abrufbar ist, Maßstäbe gesetzt und die Erforschung der Papyri und der Villa anschaulich einem breiteren (Laien)Publikum nahegebracht. ${ }^{204}$ Die Produktion wurde einige Jahre später auch für arte ins Deutsche übersetzt und im TV ausgestrahlt. Da die Experimente zum virtual unrolling und HSI (ab 2015) in bekannten naturwissenschaftlichen Journals publiziert wurden, kamen viele Nachrichtenagenturen auf die Protagonisten $\mathrm{zu}^{205}$ Auch die Konkurrenzsituation der Teams wurde thematisiert, etwa mit großer Reichweite in einem Beitrag für CBS 60 minutes (USA-2017). ${ }^{206}$ Insbesondere italienische Medien, darunter große Zeitungen, ${ }^{207}$ haben in den letzten Jahren öfters über die Papyri und die Anwendung neuer Techniken berichtet, wobei auch englischsprachige Medien zunehmend Interesse zeigen. Auch in Deutschland lösten die Herkulanischen Papyri in den letzten zwei Jahren ein gewisses Medienecho aus, wobei nicht nur die technischen Entwicklungen, sondern auch der Inhalt der Papyri und die philologische Arbeit der Papy-

203 Papyrologische Ressourcen unter https://www.herculaneum.ox.ac.uk/papyri/onlineresources.

204 Siehe Anm. 116 (auf youtube abrufbar). Angaben zum Film: Produced, Written and Directed by Julie Walker - a KBYU/BYU Television Production - 2003.

205 Vgl. Fleischer, „Report about Congress“ (wie Anm. 139).

206 www.cbsnews.com/news/herculaneum-scrolls-can-technology-unravel-the-secretssealed-by-mt-vesuvius-2000-years-ago/.

207 Etwa am 21.2.2021 im Corriere della Sera - La lettura (L'intelligenza artificiale legge i papiri di Ercolano). 
rologen thematisiert wurden. ${ }^{208}$ Die 2019 im Rahmen der Sonderausstellung in der Getty-Villa (Malibu) zu den Herkulanischen Papyri gehaltenen Vorträge sowie etliche Videos zum Thema virtual unrolling erfreuen sich im Internet wachsender Beliebtheit. ${ }^{209}$ Die für ein so kleines Fach vergleichsweise große mediale Aufmerksamkeit wird mit etwaigen (Zwischen)Erfolgen des virtual unrolling wohl nochmals steigen. Die meisten Papyrologen opfern gerne etwas Zeit, um „public outreach“ zu betreiben und die Einzigartigkeit der Herkulanischen Papyri und die Herausforderungen an die Papyrologen im öffentlichen Bewusstsein stärker zu verankern.

\subsection{Aktuelle Projekte, Entwicklungen und Orte der Forschung}

Das bereits erwähnte ERC-Projekt GreekSchools ${ }^{210}$ unter Ranocchia wird in den nächsten Jahren (2021-2025) neben dem Erstellen einer innovativen Komplett-

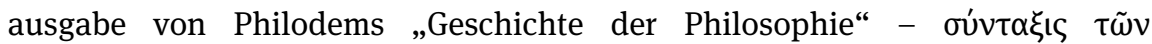
$\varphi \iota \lambda o \sigma o ́ \varphi \omega v$ (siehe 15.1) diverse Techniken an bereits entrollten Papyri erproben (siehe 10.4). Ich leite gegenwärtig das DFG-Projekt „Philodems Geschichte der Akademie (Index Academicorum)“, an dessen Ende eine Neuausgabe des Index Academicorum unter Einbezug neuer Techniken stehen soll (siehe 15).

An der Universität Würzburg ist gerade ein großes Zentrum für Digitalität und Philologie „Kallimachos“ im Aufbau begriffen, ${ }^{211}$ unter dessen Dach auch die Erforschung der Herkulanischen Papyri bzw. ihre digitale Integration weiter vorangetrieben werden könnte. Bis 2019 lief als Teilprojekt des Zentrums das von Essler betreute Anagnosis-Projekt, welches als Ziel die automatisierte Verknüpfung zwischen Transkriptionen von Papyri und Schriftzeichen der dazuge-

208 Vgl. etwa Hannes Hoffmann, „Die Hoffnung auf das Unlesbare,“ Spektrum 2019 https://www.spektrum.de/news/die-hoffnung-auf-das-unlesbare/1687132); Ulf von Rauchhaupt, Verkohlte Wörter (wie Anm. 1), (2021) - online: https://www.faz.net/aktuell/wissen/verkohltewoerter-zwei-schriftrollen-aus-der-villa-dei-papiri-17179615.html; ferner Deutschlandfunk vom 18.3.2021 („Vom Vesuv begraben - Würzburger Forscher entziffert verkohlte Texte aus Antike“) und wissenschaft.de/damals.de vom 25.3.2021 („Philosophen-Manuskript aus der Vesuvasche“ https://www.wissenschaft.de/geschichte-archaeologie/philosophen-manuskript-aus-der-vesuvasche/).

209 Vorträge von David Blank, Richard Janko und Brent Seales vom 19.10.2019 („Reading the Herculaneum Papyri: Yesterday, Today, Tomorrow“ - auf youtube abrufbar).

210 Siehe Anm. 124.

211 www.uni-wuerzburg.de/zpd/startseite/. Das Projekt führt Geisteswissenschaftler und Informatiker in einem regionalen Digital-Humanities-Zentrum zusammen. Der Bau des Zentrums soll bis 2022 fertiggestellt sein. 
hörigen Bilddateien hatte und papyrologische Bilddatenbanken mit der Volltextdatenbank DCLP (siehe 13.2.3) verbinden sollte..$^{212}$

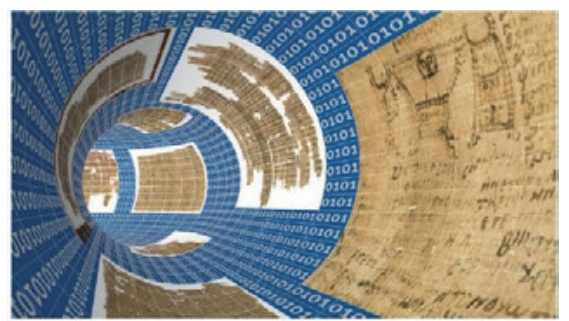

ANAGNOS15

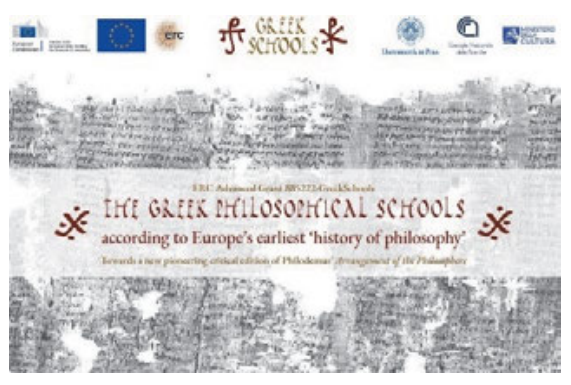

Abb. 51 und 52: Projekte Anagnosis und GreekSchools

Allgemein wird in puncto Digitalität versucht, Synergieeffekte mit anderen Teilbereichen der Papyrologie zu generieren (siehe etwa DCLP) und, soweit möglich und wo sinnvoll, die „Herkulanischen tools“ in einen breiteren Kontext zu integrieren und mit Nachbardisziplinen zu verbinden. In der Gesamtschau hat die Herkulanische Papyrologie von den Möglichkeiten der Digitalisierung, Bildbearbeitungsprogrammen und neuen Bildgebungsverfahren (MSI) im Vergleich zu anderen Zweigen der Philologie oder Papyrologie aufgrund der Beschaffenheit der Papyri und den damit verbundenen Defiziten früherer Editionen wohl überproportional Nutzen gezogen.

Die Mehrzahl der Herkulanischen Papyrologen, welche von ihrer akademischen Ausbildung her fast ausnahmslos Klassische Philologen sind, stammt aus Italien und forscht ebendort, freilich allen voran an der Quelle in Neapel. Daneben arbeiten bzw. arbeiteten in den letzten Dekaden auch im englischsprachi-

212 http://www.kallimachos.de/kallimachos/index.php/Anagnosis. Ein von Kallimachos entwickelter Online-Editor soll die automatisierte Verknüpfung von Text und Abbildung auf Buchstabenebene bei den Papyri ermöglichen, was insbesondere für paläographische Vergleichszwecke und für graphische Rekonstruktionen ausgenutzt werden soll. Zum Projekt siehe auch Reggiani, Digital Papyrology (wie Anm. 136),154-156 und Holger Essler und Vincenzo Damiani, „Anagnosis. Automatisierte Buchstabenverknüpfung von Transkript und Papyrusabbildung," in Altertumswissenschaften in a Digital Age: Egyptology, Papyrology and beyond; proceedings of a conference and workshop in Leipzig, November 4-6, 2015 (hg. von Monica Berti und Franziska Naether; Leipzig, 2016) und Ast und Essler, „Anagnosis“ (wie Anm. 195), 71-73. 
gen Raum etwa ein Dutzend Wissenschaftler vertieft an den Herkulanischen Papyri. Auch einzelne Forscher aus anderen Ländern widmen sich den Papyri. In Deutschland beschäftigen sich bedauerlicherweise nur wenige Wissenschaftler mit der Edition von Herkulanischen Papyri und kaum einer forscht exklusiv oder vornehmlich an den Herkulanischen Texten. Neben Würzburg um den früheren Gräzistik-Lehrstuhlinhaber Michael Erler und seinen Nachfolger Jan Stenger ist als einzige deutsche Zufluchtsstätte der Musa Herculanensis die Universität Köln um Jürgen Hammerstaedt geblieben, der sich viele Verdienste um die Papyri erworben hat und mehrere „Herkulanische“ Gastwissenschaftler betreute. ${ }^{213}$ Etliche Forschungsaktivitäten wurden formell oder faktisch vom CISPE koordiniert, während auch stets Wissenschaftler mit nur loser oder keiner Verbindung zum CISPE an den Papyri forschten. Die Ergebnisse der Herkulanischen Papyrologie, sofern sie von allgemeinem Interesse sind, werden in der Klassischen Philologie, aber auch in der Philosophie, Theologie und Alten Geschichte rege rezipiert.

213 Hammerstaedt wandte sich in den letzten beiden Dekaden auch verstärkt der Inschrift von Oinoanda zu. 